\title{
Metastatic Mediastinal Lymph Node from an Unidentified Primary Papillary Thyroid Carcinoma Diagnosed by Endobronchial Ultrasound-Guided Transbronchial Needle Aspiration
}

\author{
Anson Chow ${ }^{1,2}$, Masahide Oki ${ }^{1}$, Hideo Saka ${ }^{1}$, Suzuko Moritani ${ }^{3}$ and Noriyasu Usami ${ }^{4}$
}

\begin{abstract}
Real-time endobronchial ultrasound-guided transbronchial needle aspiration (EBUS-TBNA) with a dedicated EBUS bronchoscope has been reported as a minimally invasive and accurate method for sampling specimens from mediastinal and hilar lesions. Using this method, not only cytologic but also histologic specimens, which provide valuable information for a definitive diagnosis, can often be obtained. We report a case of an enlarged metastatic mediastinal lymph node from an unidentified primary papillary thyroid carcinoma that was accurately diagnosed by histological and immunohistochemical examination of tissue obtained by EBUS-TBNA.
\end{abstract}

Key words: endobronchial ultrasound, transbronchial needle aspiration, mediastinal lymph node, metastasis, thyroid carcinoma

(Inter Med 48: 1293-1296, 2009)

(DOI: 10.2169/internalmedicine.48.2212)

\section{Introduction}

In the absence of an identifiable primary site of a tumor, an enlarged mediastinal lymph node due to metastasis is occasionally encountered as the main clinical presentation. In such cases, biopsy of the mediastinal lymph node is a rapid and accessible way to obtain a definite pathological diagnosis. With recent advances in immunohistochemical studies using various antibodies, the tumor lineage or even a specific tumor can be accurately identified provided adequate histological tissue is obtained (1).

Endobronchial ultrasound-guided transbronchial needle aspiration (EBUS-TBNA), which is performed by confirming the position of a dedicated echogenic needle tip under EBUS imaging during the needle aspiration procedure, is a relatively new, minimally invasive and accurate bronchoscopic procedure for sampling mediastinal lesions (2-5). Although various investigators have reported its usefulness for evaluating mediastinal metastasis from proven or suspected malignancies such as lung cancer (2-4) or other intra- or extrathoracic malignancies (6), few reports have addressed its utility in the initial diagnosis of malignancies of unknown origin. We report a case of an enlarged metastatic mediastinal lymph node from an unidentified primary papillary thyroid carcinoma that was accurately diagnosed by cytological, histological and immunohistochemical examination of specimens by EBUS-TBNA.

\section{Case Report}

A 56-year-old woman complained of sudden onset of hoarseness of voice with no palpable neck nodule or palpable lymph node on physical examination. She had no significant past medical history or any history of malignancy. Chest radiograph showed mild widening of the upper mediastinum. Chest computed tomography (CT) revealed an enlarged mediastinal lymph node in the right highest mediasti-

\footnotetext{
${ }^{1}$ Department of Respiratory Medicine, Nagoya Medical Center, Nagoya, ${ }^{2}$ Department of Respiratory Medicine, Ruttonjee \& Tang Shiu Kin Hospital, Hong Kong, People's Republic of China, ${ }^{3}$ Department of Pathology, Nagoya Medical Center, Nagoya and ${ }^{4}$ Department of Thoracic Surgery, Nagoya University School of Medicine, Nagoya

Received for publication February 25, 2009; Accepted for publication April 13, 2009

Correspondence to Dr. Masahide Oki, masahideo@aol.com
} 


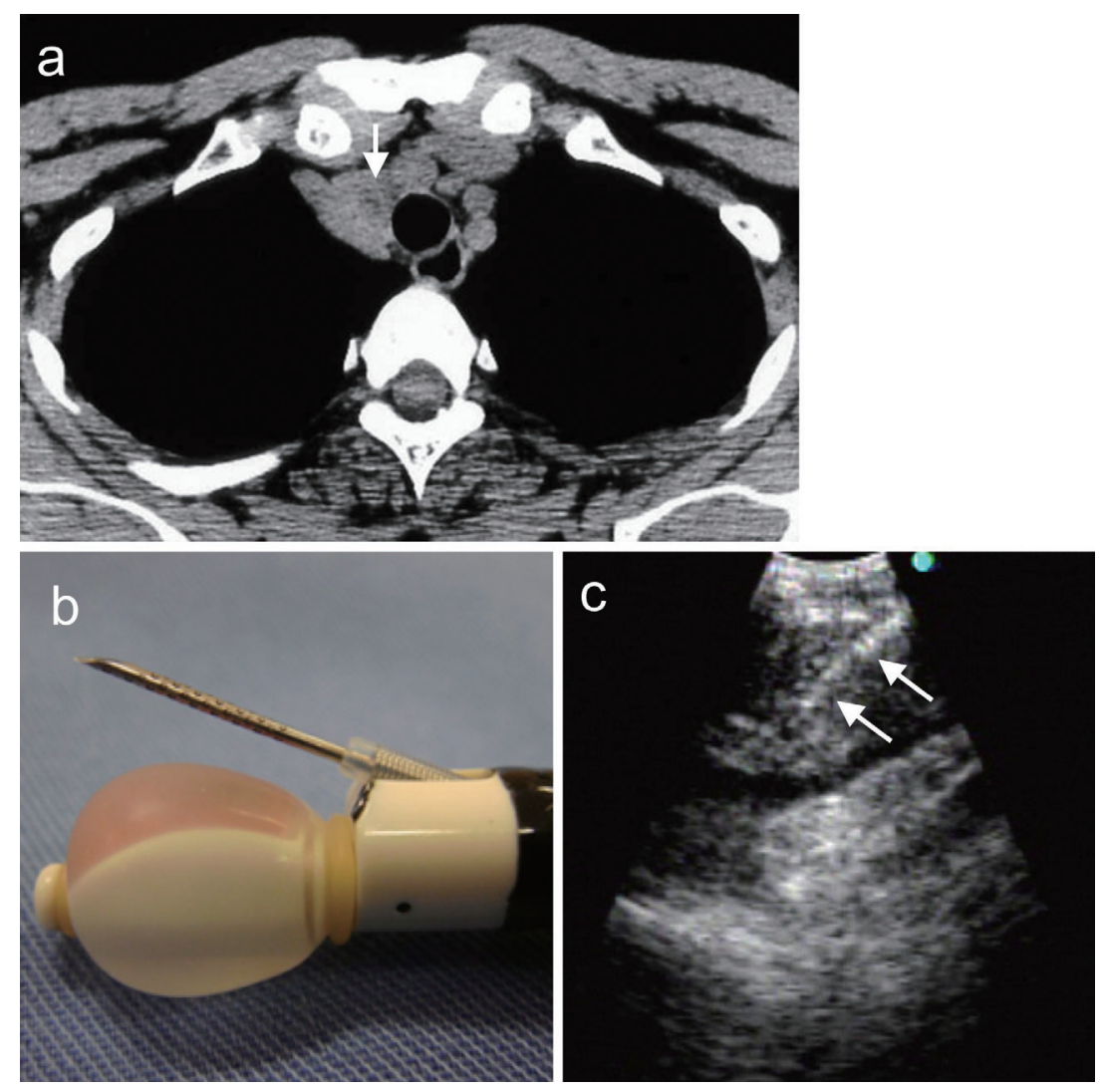

Figure 1. a) Chest computed tomography showing the right highest mediastinal lymph node (arrow) measuring $20 \mathrm{~mm}$. b) Tip of the ultrasonic bronchoscope with inflated balloon (BF-UC260FOL8; Olympus) and dedicated echogenic 22-gauge needle (NA-201SX-4022; Olympus). c) EBUSTBNA performed with real-time visualized needle (arrows) within the lymph node.

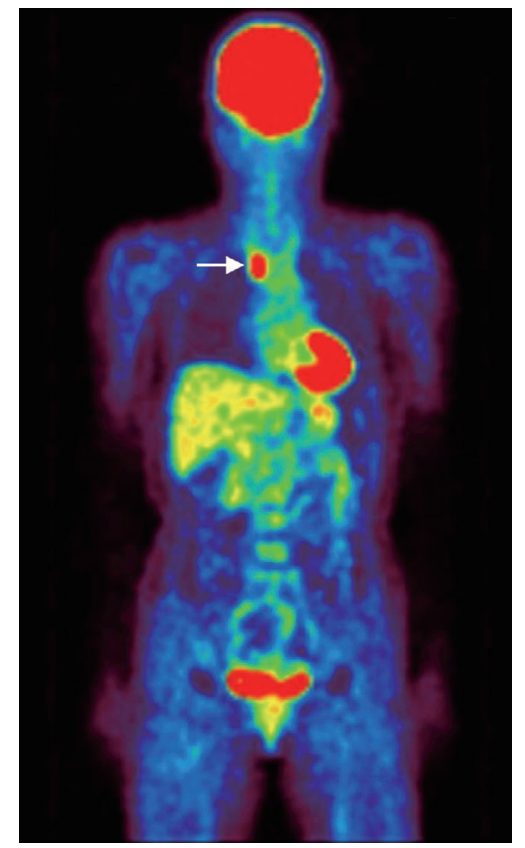

Figure 2. Fluorodeoxyglucose positron emission tomography showing a hypermetabolic lesion in the right highest mediastinal region (arrow).

nal region [ATS station 1R, (7)] measuring $20 \mathrm{~mm}$
(Fig. 1a). Fluorodeoxyglucose positron emission tomography was positive in the corresponding region, suggesting possible malignancy, but no abnormal uptake was found in other sites including the thyroid gland (Fig. 2). She was then referred to our hospital for further evaluation.

Standard conventional flexible bronchoscopy was first performed to examine the tracheobronchial tree, which revealed only right vocal cord palsy. No other endobronchial lesion was seen. A linear array ultrasonic bronchoscope using a built-in 7.5 MHz linear probe with dedicated 22-gauge needle (BF-UC260F-OL8; Olympus, Tokyo, Japan, Fig. 1b) was subsequently used to perform ultrasonic examination and EBUS-TBNA. All mediastinal and hilar lymph node stations were systematically imaged. An enlarged lymph node on the right highest mediastinal station (station 1R) was clearly visualized on EBUS imaging. EBUS-TBNA under direct real-time guidance (Fig. 1c) was performed on this enlarged lymph node. Both cytological and histological specimens were successfully collected for pathological examination. Cytological findings revealed intranuclear cytoplasmic inclusions (Fig. 3a), nuclear grooves and psammoma bodies (Fig. 3b) on Papanicolaou stain, while histological finding showed carcinoma cells with ground glass nuclei (Fig. 3c). Both cytological and histological findings were suggestive of papillary thyroid carcinoma. Immunohis- 

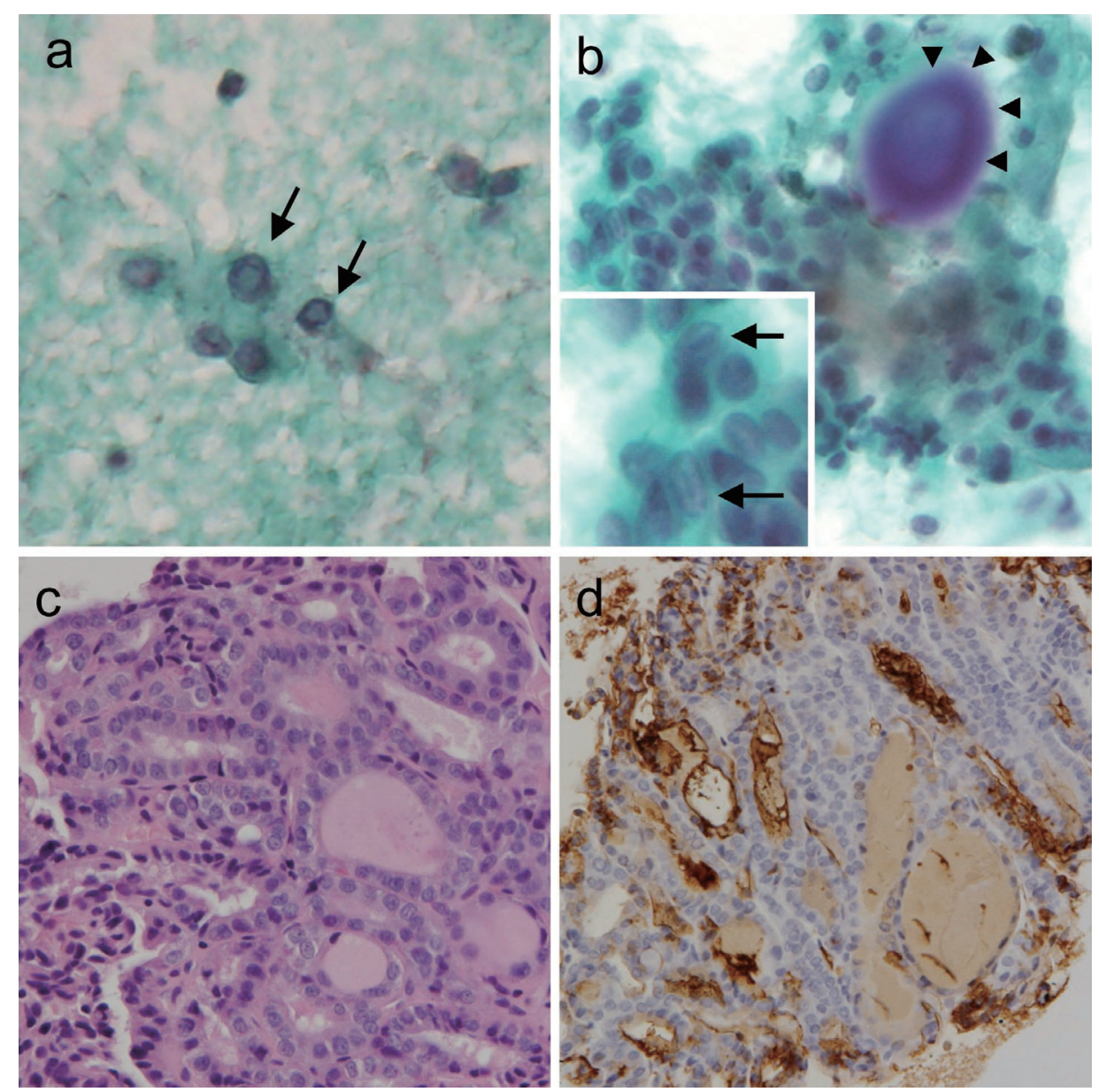

Figure 3. a) Cytological specimen showing intranuclear cytoplasmic inclusions (arrows). (Papanicolaou stain, original $\times 400$ ). b) Cytological specimen showing nuclear grooves (arrows) and psammoma bodies (arrowheads). (Papanicolaou stain, original $\times 400)$. c) Histological specimen of lymph node obtained by EBUS-TBNA, showing carcinoma cells with ground glass nuclei. (Hematoxylin and Eosin staining, original $\times 400$ ). d) Immunohistochemical study for thyroglobulin showing positive brownish staining of tumor cell suggesting thyroid gland origin.

tochemical study was also performed on a histological specimen that was positively stained with anti-thyroglobulin antibody (DakoCytomation, Denmark, clone: DAK-Tg6, Fig. 3d). The primary tumor origin of thyroid gland was then confirmed.

Later the patient underwent an operation in which a 13$\mathrm{mm}$ primary thyroid tumor was found over the left lobe of the gland. Total thyroidectomy with lymph node dissection was done. During the operation the right highest mediastinal metastatic lymph node was found to invade the surrounding brachiocephalic artery and was incompletely resected. Histological examination of the resected specimens confirmed well-differentiated papillary thyroid carcinoma with mediastinal lymph node metastasis, which corresponded to our pre-operative tissue diagnosis by EBUS-TBNA. Later the patient underwent and completed post-operative radiotherapy on the right upper paratracheal region with regular follow-up in an outpatient clinic in stable condition.

\section{Discussion}

Despite its rarity, mediastinal lymph node metastasis from papillary thyroid carcinoma is a well known clinical condition (8). Nevertheless, with time, both primary thyroid tumor and metastatic mediastinal lymph node may eventually lead to the invasion of surrounding organs, such as the upper airway, which can become a life-threatening condition. Thus, earlier surgical excision of the tumor and involved lymph node can help to prevent further distant metastases or subsequent upper airway obstruction. Furthermore, earlier diagnosis and treatment of malignancy can definitely help to reduce patient anxiety and stress due to initial uncertainty of primary tumor origin and may also offer survival benefit.

To date, the evaluation of the mediastinal lymph nodes has been a challenging diagnostic problem, because they adjoin mediastinal structures such as the great vessels. Accurate mediastinal lymph node sampling is usually difficult by conventional TBNA using a standard bronchoscope by pass- 
ing the needle through the airway wall and into a lymph node guided by static CT scan or fluoroscopy. As it is a blind procedure, the successful sampling rate varies widely between 33 and 78\% (9). On the other hand, mediastinoscopy or video-assisted thoracotomy is considered the gold standard for tissue sampling of the mediastinum, but it is an invasive procedure requiring general anesthesia and is not without risk (10).

Direct real-time EBUS-TBNA is a relatively new and safe method for sampling mediastinal tissue and the diagnostic yield is very high, with a reported rate of greater than $90 \%$ (11). Doppler ultrasound can be used to identify vessels as necessary to minimize unintended puncture of blood vessels. Currently, EBUS-TBNA can be safely performed under local anesthesia and in an outpatient setting. In the present case study, both cytological and histological samples were successfully obtained and revealed characteristic features of papillary thyroid carcinoma, while immunohistochemical study revealed that the tissue core was positively stained with anti-thyroglobulin antibody, confirming the origin of primary thyroid tumor. The patient well tolerated the proce- dure without any complications.

To date, a few cases with a recurrent papillary thyroid carcinoma successfully diagnosed by EBUS-TBNA have been reported $(12,13)$. Since the launch of EBUS-TBNA, we performed EBUS-TBNA on 302 patients in our hospital from March 2004 to February 2009. Three patients in all were newly found to have a metastatic mediastinal lymph node from papillary thyroid carcinoma by EBUS-TBNA, including the present patient. Two of them presented with isolated vocal cord palsy without palpable thyroid nodule or neck lymph node or initial suspicion of thyroid cancer, while the other one with confirmed recurrent papillary thyroid carcinoma was referred for investigation of an enlarged mediastinal lymph node. All of the EBUS-TBNA findings of these 3 patients were confirmed by post-operative pathological examination.

Thus, in the evaluation of a papillary thyroid carcinoma patient with mediastinal adenopathy, EBUS-TBNA is an excellent and safe tool to obtain adequate histological nodal tissue for examination, in order to achieve a rapid and definitive pathological diagnosis.

\section{References}

1. Fischer S, Asa SL. Application of immunohistochemistry to thyroid neoplasms. Arch Pathol Lab Med 132: 359-372, 2008.

2. Yasufuku K, Chiyo M, Sekine Y, et al. Real-time endobronchial ultrasound-guided transbronchial needle aspiration of mediastinal and hilar lymph nodes. Chest 126: 122-128, 2004.

3. Yasufuku K, Chiyo M, Koh E, et al. Endobronchial ultrasound guided transbronchial needle aspiration for staging of lung cancer. Lung Cancer 50: 347-354, 2005.

4. Herth FJ, Eberhardt R, Vilmann P, Krasnik M, Ernst A. Real-time endobronchial ultrasound-guided transbronchial needle aspiration for sampling mediastinal lymph nodes. Thorax 61: 795-798, 2006.

5. Oki M, Saka H, Kitagawa C, et al. Real-time endobronchial ultrasound-guided transbronchial needle aspiration is useful for diagnosing sarcoidosis. Respirology 12: 863-868, 2007.

6. Nakajima T, Yasufuku K, Iyoda A, et al. The evaluation of lymph node metastasis by endobronchial ultrasound-guided transbronchial needle aspiration: crucial for selection of surgical candidates with metastatic lung tumors. J Thorac Cardiovasc Surg 134: 1485-1490, 2007.

7. Mountain CF, Dresler CM. Regional lymph node classification for lung cancer staging. Chest 111: 1718-1723, 1997.
8. Carling T, Udelsman R. Thyroid tumors. In: Cancer: Principles \& Practice of Oncology. 8th ed. DeVita VT Jr, Lawrence TS, Rosenberg SA, Eds. Lippincott-Raven, Philadelphia, 2008: 1663-1682.

9. Holty JE, Kuschner WG, Gould MK. Accuracy of transbronchial needle aspiration for mediastinal staging of non-small cell lung cancer: a meta-analysis. Thorax 60: 949-955, 2005.

10. Detterbeck FC, Jantz MA, Wallace M, Vansteenkiste J, Silvestri GA; American College of Chest Physicians. Invasive mediastinal staging of lung cancer: ACCP evidence-based clinical practice guidelines (2nd edition). Chest 132(Suppl): 202S-220S, 2007.

11. Yasufuku K, Nakajima T, Fujiwara T, et al. Role of endobronchial ultrasound-guided transbronchial needle aspiration in the management of lung cancer. Gen Thorac Cardiovasc Surg 56: 268-276, 2008.

12. Sakairi Y, Yasufuku K, Iyoda A, et al. A solitary metastatic lung tumor from thyroid papillary carcinoma diagnosed by endobronchial ultrasound-guided transbronchial needle aspiration (EBUSTBNA): report of a case. Surg Today 38: 46-48, 2008.

13. Diaz J, Chawla M, Simoff M. Endobronchial ultrasound-guided transbronchial needle aspiration in the diagnosis of metastatic thyroid cancer. J Bronchol 16: 70-71, 2009.

(C) 2009 The Japanese Society of Internal Medicine http://www.naika.or.jp/imindex.html 